\title{
O USO DE GEOTECNOLOGIAS E TICS NO ENSINO-APRENDIZAGEM DE CIÊNCIA DO SOLO
}

\author{
$\underline{\text { Ramon Oliveira Santos }}{ }^{1}$; Alexandre dos Santos Silva ${ }^{2}$; Roneíse de Jesus Lima ${ }^{3}$, Joselisa \\ Maria Chaves ${ }^{4}$ \\ 1. Bolsista PIBIC/CNPq, Graduando em Agronomia, Universidade Estadual de Feira de Santana, \\ e-mail: osantos.ramon@gmail.com \\ 2. Discente do curso de Sistemas de Informação da Universidade Salvador, \\ e-mail: alexandhre.developer@gmail.com \\ 3. Bolsista Probic, Graduanda em Geografia, Universidade Estadual de Feira de Santana, \\ e-mail: isedelima@gmail.com \\ 3. Orientadora, Departamento de Ciências Exatas, Universidade Estadual de Feira de Santana, \\ e-mail: joselisa@uefs.br
}

PALAVRAS-CHAVE: Geoprocessamento; Agronomia; Geociências.

\section{INTRODUÇÃO}

O solo é uma mistura de compostos minerais e orgânicos da superfície terrestre que funciona como substrato para o desenvolvimento das plantas (MOREIRA, 2007). Nos dias atuais, é crescente a busca de soluções que busquem proteger este recurso natural, pois o manejo inadequado e a falta de informação são os grandes responsáveis pela degradação do solo.

O ensino de Ciência do Solo nas universidades ainda segue um modelo clássico que não estimula os alunos a buscar mais informações sobre esse tema. O curso de Agronomia possui disciplinas como Geociências, Pedologia, Física e Química do Solo, que permitem um ensino mais próximo da prática, abrindo assim possibilidades para caminhos de aprendizado mais dinâmico e criativo.

Geotecnologia é a arte de estudar a superfície da terra e adaptar as informações às necessidades dos meios físicos, químicos e biológicos (SILVA, 1999). A geotecnologia surge então, como uma forma alternativa e inovadora de ensino de diferentes ciências, que podem ser aplicadas ao estudo do solo, pois tem o poder de unir conceitos de cartografia, geografia e agronomia.

Além disso, as "novas tecnologias" alteraram a forma de relacionamento do indivíduo com o outro e com si próprio (ALMENARA, 1996). Segundo Mendes (2008) as Tecnologias da Informação e Comunicação (TICS) são um conjunto de recursos tecnológicos que proporcionam a automação e/ou a comunicação nos processos existentes nos negócios, no ensino e na pesquisa científica.

A inserção dos discentes no contexto da informática pode contribuir para a descoberta de um caminho em que o uso de programação nos computadores do laboratório pode se tornar uma nova forma de aprender Ciência do solo, despertando a curiosidade do aluno para uma área que é pouco explorada dentro da Agronomia.

O presente trabalhou buscou fazer um levantamento bibliográfico de todo material teórico necessário para a criação de práticas interativas, uma vez que o eixo teoria-prática é indissociável, visando à elaboração de materiais de ensino criativos e inovadores que facilitarão o ensino-aprendizagem de Ciência do Solo.

\section{METODOLOGIA}

A metodologia utilizada para a criação do game pode ser observada na figura 1.

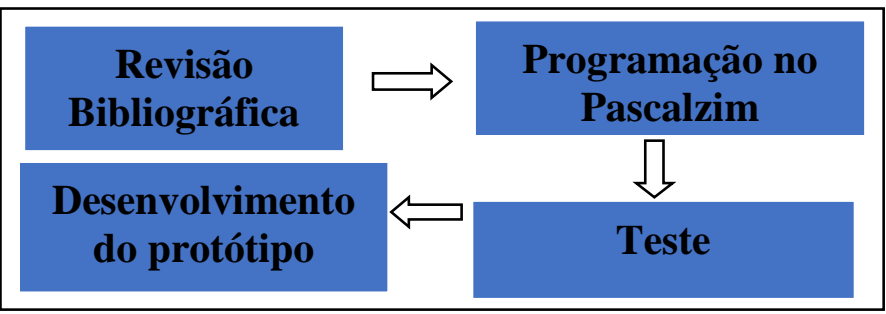

Figura1. Fluxograma metodológico da criação do game. 
Realizou-se uma pesquisa bibliográfica com temas relacionados a ciência do solo para fundamentar o game. Foram escolhidos alguns artigos, dentre eles: "História da pedologia: um resgate bibliográfico" de Carlos Roberto Espindola; "Capítulo 1: Ciência do solo evolução do conhecimento" de Egon Klamt e Dalvan José Reinert; "O ciclo das rochas na natureza" de Celso Dal Ré Carneiro et al.; e o livro "Superfície da Terra" de Arthur L. Bloom. O "Quiz - Ciência do Solo" foi programado no compilador "Pascalzim" com 31 questões no estilo verdadeiro-falso. Quando o estudante não responde corretamente "V ou F", o programa exibe uma mensagem com a correção da afirmação, chamando a atenção para o seu erro, já quando o estudante acerta, o programa exibe uma mensagem parabenizando-o. Há ainda uma contagem de pontos, "score", para os usuários terem noção de quantas questões acertaram.

Já a etapa final foi o desenvolvimento do protótipo de alta fidelidade, ou seja, o game em uma linguagem mais avançada, C\#, programado para plataforma desktop.

Para a criação da cartilha sobre os principais solos do Nordeste foi feita uma revisão bibliográfica de artigos, livros e apostilas sobre solos, para então ser elaborada um material didático que reunisse o máximo de informações sobre solos.

Para a criação das oficinas sobre "Solos e agricultura familiar" e "Aplicações de geotecnologias no estudo de solos" foi feita uma leitura de artigos e livros sobre os temas, e adotou-se na primeira a proposta do uso de artigo científico como recurso didático no ensino superior. Nesta primeira oficina, também foi apresentado o game "Quiz - Ciência do solo" para os discentes. Na segunda oficina, ocorreu no primeiro momento uma discussão teórica sobre os assuntos e depois fez-se uma atividade prática com os discentes nos computadores do laboratório.

\section{ANÁLISE E DISCUSSÃO DOS RESULTADOS}

O primeiro resultado obtido foi a cartilha de autopreenchimento sobre os principais solos do Nordeste, intitulada "Viajando pelos solos do Nordeste". A cartilha traz capítulos sobre perfil do solo, morfologia do solo e caracterização ambiental e descrição de solos do Nordeste. É uma cartilha que poderá ser utilizada em sala de aula nas disciplinas de Ciência do solo, facilitando assim a compreensão dos solos da região.

O segundo resultado obtido foi a criação do jogo eletrônico "Quiz - Ciência do solo". O game educativo foi primeiramente criado no "Pascalzim", e pode ser utilizado pelo discente em sala de aula com o auxílio do docente, possibilitando aulas mais engajadas e enriquecedoras, ou até mesmo em casa para o discente reforçar os seus conhecimentos em ciência do solo.

O envolvimento do aluno com as disciplinas relacionada a solos provavelmente aumentará, uma vez que o modo como o conteúdo é apresentado - nesse caso, por um game educativo tem relação direta com a motivação deste em ter autonomia, proatividade e agilidade de pensamento para aumentar sua "bagagem" sobre ciência do solo (KESSLER et al., 2010).

As disciplinas da ciência do solo - geociências, pedologia, física do solo, química e fertilidade do solo - oferecidas na Universidade Estadual de Feira de Santana (UEFS) são extremamente importantes para uma formação sólida de engenheiros agrônomos. Desta forma, buscar instrumentos variados como auxílio e fonte de aprendizagem é uma saída para o ensino dessa área de estudo.

Os jogos eletrônicos, ou games, tem um grande potencial educativo que, se bem utilizado, pode proporcionar um ganho de conhecimento significativo aos estudantes de nível superior. É importante entender que a o jogo deve valorizar não só a fundamentação teórica, como também alternativas para a formação de profissionais com capacidade analítica, consciente e altruísta. Assim, games tem o poder de preparar para o mundo, além de dinamizar as aulas e aliviar a ansiedade dos discentes (FRIEDMANN, 1998).

No "Quiz - Ciência do Solo" (figura 1), a segunda tela (figura 2) traz informações sobre o objetivo geral do jogo, instruções sobre como jogar, e o componente motivador "Cada pergunta vale 2 pontos. Sucesso!!”, que faz o aluno ficar mais atento, estimulando o desenvolvimento total do discente (RIZZO, 1996). 


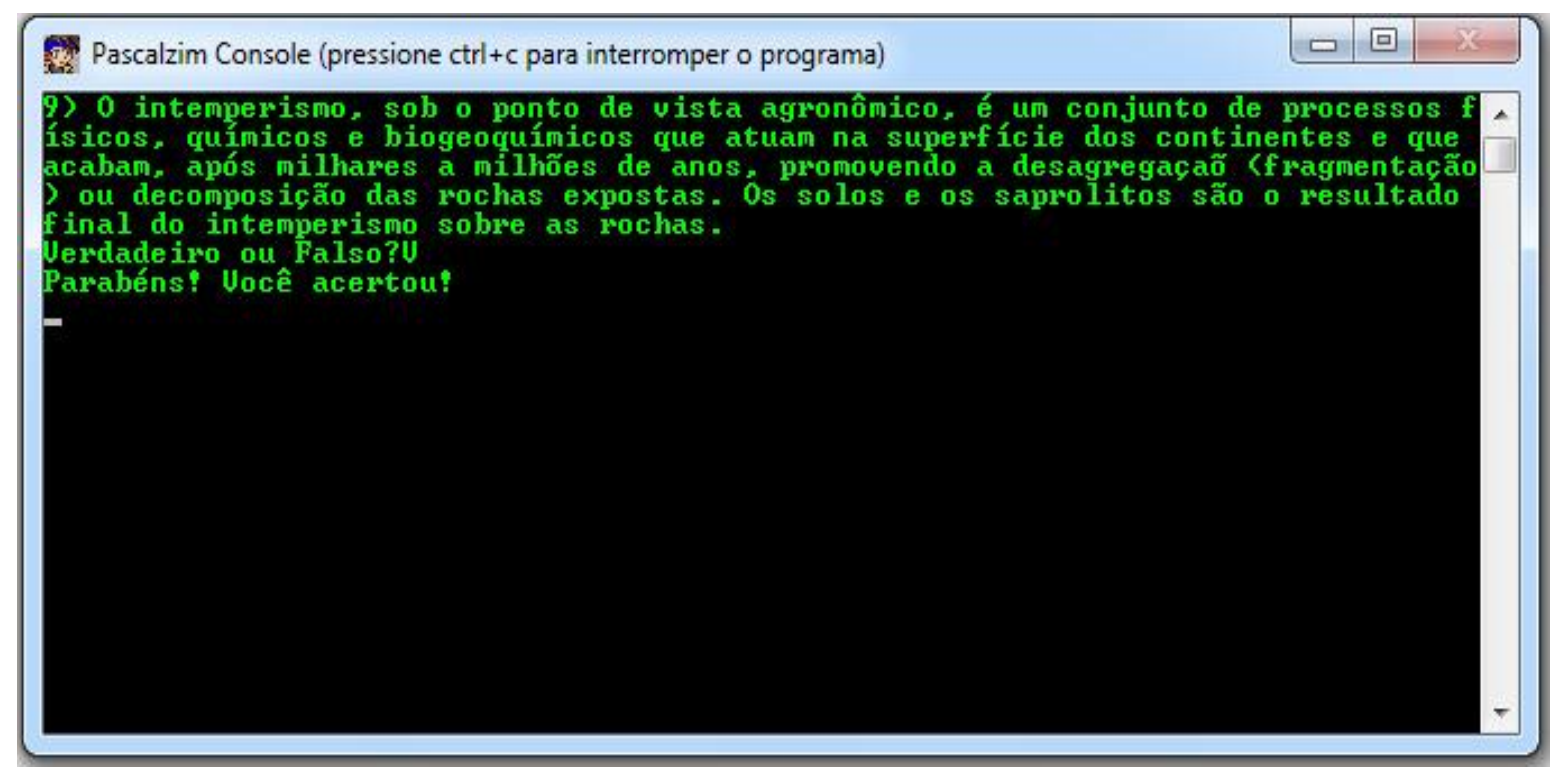

Figura 2. Pergunta 9 do Quiz, exemplo de resposta dada e parabenização.

Posteriormente, seguiu-se para a etapa de desenvolvimento do protótipo de alta fidelidade (figura 3), onde o jogo foi programado em uma linguagem mais avançada, C\#, testado e aplicado com os alunos do curso agronomia na oficina sobre solos e agricultura familiar.

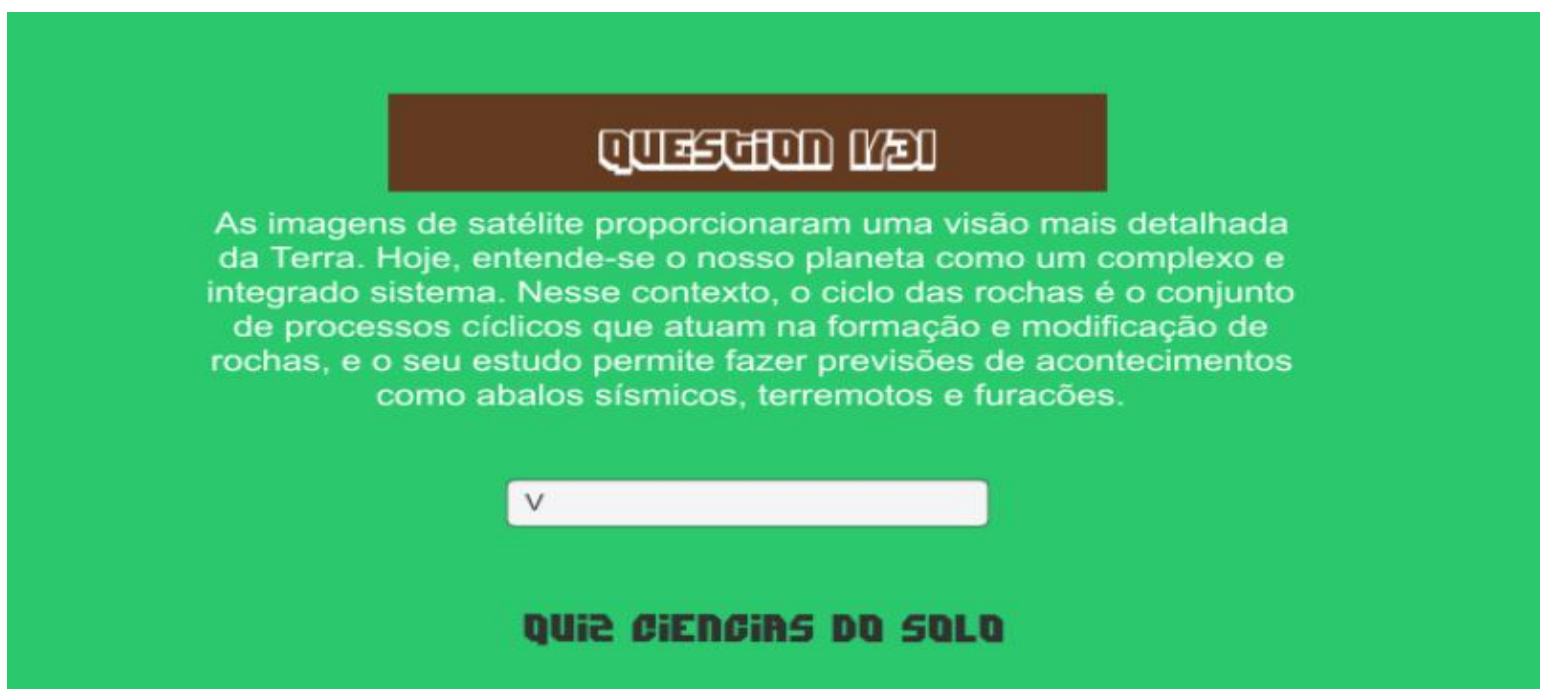

Figura 3. Pergunta 1 do Quiz Ciências do Solo

O terceiro resultado da pesquisa foi a oficina intitulada "Aplicações de Geotecnologias no estudo de solos" que abordou aspectos teóricos e práticos sobre Sistemas de Informações Geo-referenciadas e Sensoriamento Remoto no estudo de solos. Esta foi aplicada para alunos do curso de Geografia e Agronomia, e ministrada com o apoio de uma bolsista do curso de Geografia. Os alunos mostraram-se envolvidos e curiosos na parte prática, que foi a caracterização de curvas espectrais de solo no software ENVI Classic.

O último resultado do trabalho foi a oficina sobre "Solos e Agricultura Familiar", aplicada para alunos do curso de Agronomia e que teve duração de 2 horas. Discutiu-se sobre essa temática que é o foco do curso de Agronomia da UEFS e utilizou-se o artigo científico "Práticas produtivas da agricultura familiar: um estudo no município de espigão D'oeste (RO)" para fundamentar a discussão.

Visto que o desenvolvimento da habilidade de comunicação oral é extremamente importante na vida dos universitários, é preciso realizar atividades que promovam a prática dessa habilidade. Além disso, sabe-se que no Brasil o uso de artigos científicos como ferramenta 
auxiliar de ensino ainda é deficiente (MASSI et al., 2009). Trazer para a sala de aula um recurso como esse pode melhorar o ensino-aprendizagem dos conteúdos específicos das disciplinas do curso de Agronomia, aperfeiçoar habilidades de comunicação oral e escrita em linguagem científica e fazer os alunos se habituarem com o desenvolvimento do conhecimento e pesquisa científica. A atenção para essas questões específicas é válida, pois os alunos precisarão dessas habilidades em sua atuação profissional, principalmente se seguirem a carreira acadêmica.

O ensino-aprendizagem de conteúdos de Agronomia foi o objetivo principal da proposta, a leitura e a compreensão dos artigos envolveram, necessariamente, o estudo de alguns conceitos de solos e agricultura familiar. Assim, as seguintes solicitações e questões propostas aos estudantes se mostraram como potenciais para despertar o interesse dos alunos pelo aprendizado de conteúdos específicos: qual foi o conceito de solo utilizado no trabalho? O autor utilizou mais de um conceito? O autor abordou propriedades físicas e químicas do solo? Apresentem uma análise dos gráficos, tabelas e imagens presentes no artigo.

No momento final, os alunos receberam um feedback positivo sobre suas contribuições na oficina e comentaram sobre a relevância da proposta do uso de artigos científicos para a sua formação profissional. Além disso, pode-se perceber a interação gerada ao utilizar o Quiz Ciência do Solo, pois todos sentiram-se desafiados e estimulados a acertar as questões, buscando atingir uma maior pontuação no jogo.

\section{CONSIDERAÇÕES FINAIS}

O desenvolvimento deste trabalho permitiu entender o quanto é necessário a utilização de ferramentas diferenciadas para dinamizar o ensino-aprendizagem de Ciência do solo. Jogos educativos, cartilhas bem produzidas e oficinas com temas referentes a essa área de estudo tornam-se alternativas relevantes para melhorar a apreensão de conhecimentos pelos discentes. Uma vez que, na sociedade atual, as mudanças estão acontecendo em ritmo acelerado, é preciso romper com modelos arcaicos de educação e ousar na metodologia de ensino-aprendizagem, pois só assim será possível formar profissionais eficientes quanto a resolução de problemas.

\section{REFERÊNCIAS}

MOREIRA, M.A. Fundamentos de Sensoriamento Remoto e metodologias de Aplicação, $3^{\text {a }}$ ed. Viçosa: UFV, 2007, 320p.

SILVA, A. B. Sistemas de Informações Geo-referenciadas: conceitos e fundamentos. Campinas: Unicamp, 1999. 236 p. (Coleção Livro-Texto).

ALMENARA, Julio Cabero(1996). Nuevas tecnologias, comunicacion y educación. Edutec. Revista Electrónica De Tecnología Educativa. Núm. 1. Febrero . Palma De Mallorca

MENDES, Alexandre. TIC - Muita gente está comentando, mas você sabe o que é?. IMasters, 2008. Disponível em: < http://imasters.com.br/artigo/8278/gerencia-de-ti/tic-muitagente-estacomentando-mas-voce-sabe-o-que-e/>. Acesso em: 25 ago. 2013.

KESSLER, M. C.; de Paula, C. G.; Albé, M. H.; Manzini, N.; Barcellos, C.; Carlson, R.; Marcon, D.; Kehl, C., (2010). "Impulsionando a aprendizagem na universidade por meio de jogos educativos digitais”, In: XXI Simpósio Brasileiro de Informática na Educação (SBIE), João Pessoa - PB, Brasil.

FRIEDMANN, Adriana. A evolução do brincar. IN: FRIEDMANN, Adriana [ et al.]. 4. ed., São Paulo: Edições Sociais: Abrinq, 1998.

RIZZO, Gilda. Jogos inteligentes: a construção do raciocínio na escola natural. Rio de Janeiro: Bertrand Brasil, 1996.

MASSI, L.; SANTOS, GR.; FERREIRA, JQ.; QUEIROZ, SL. Artigos científicos como recurso didático no ensino superior de Química. Química Nova 32 (2), 503-510. 2009. 